Supporting Information

For

\title{
Effective Management of Nucleation and Crystallization Processes in Perovskite Formation via Facile Control of Anti-Solvent Temperature
}

Hossein Taherianfard, ${ }^{b \neq}$ Guan-Woo Kim, ${ }^{a \neq}$ Mahdi Malekshahi Byranvand, ${ }^{b}$ Kyoungwon

Choi, ${ }^{a}$ Gyeongho Kang, ${ }^{a}$ Hyuntae Choi, ${ }^{a}$ Fariba Tajabadi, ${ }^{c}$ Nima Taghavinia, ${ }^{b, d *}$ and

Taiho Park *

a Dr. Guan-Woo Kim, Kyoungwon Choi, Dr. Gyeongho Kang, Hyuntae Choi, Dr. Taiho

Park

Department of Chemical Engineering, Pohang University of Science and Technology (POSTECH), 77 Cheongam-Ro, Nam-gu, Pohang, Kyoungbuk, Korea (37673).

E-mail: taihopark@postech.ac.kr

${ }^{\mathrm{b}}$ Hossein Taherianfard, Dr. Mahdi Malekshahi Byranvand, Dr. Nima Taghavinia

Department of Physics, Sharif University of Technology, Tehran 14588, Iran.

E-mail: taghavinia@sharif.edu

${ }^{c}$ Dr. Fariba Tajabadi 
Department of Nanotechnology and Advanced Materials, Materials and Energy Research

Centre, Karaj, 31787-316, Iran.

d Dr. Nima Taghavinia

Institute for Nanoscience and Nanotechnology, Sharif University of Technology, Tehran

14588-89694, Iran

‡ These authors contributed equally to this work. 


\section{Contents}

Figure S1. UV-visible absorption spectra of the intermediate films formed at different chlorobenzene temperatures $\left(0,20,35,50\right.$, and $\left.100^{\circ} \mathrm{C}\right)$.

Figure S2. Steady-state photoluminescence of perovskites on tin oxide layer.

Figure S3. Photoluminescence quenching of perovskites on tin oxide layer.

Figure S4. Stability tests of each device fabricated at various temperatures: (a) short-circuit current, (b) open-circuit voltage, (c) fill factor, (d) power conversion efficiency.

Table S1 The thickness of perovskite films formed at different chlorobenzene temperatures $(0,20$, 35,50 , and $100^{\circ} \mathrm{C}$ ).

Table S2 The average photovoltaic parameters of full devices with different chlorobenzene temperatures 


\section{Supplementary Figures and Tables}

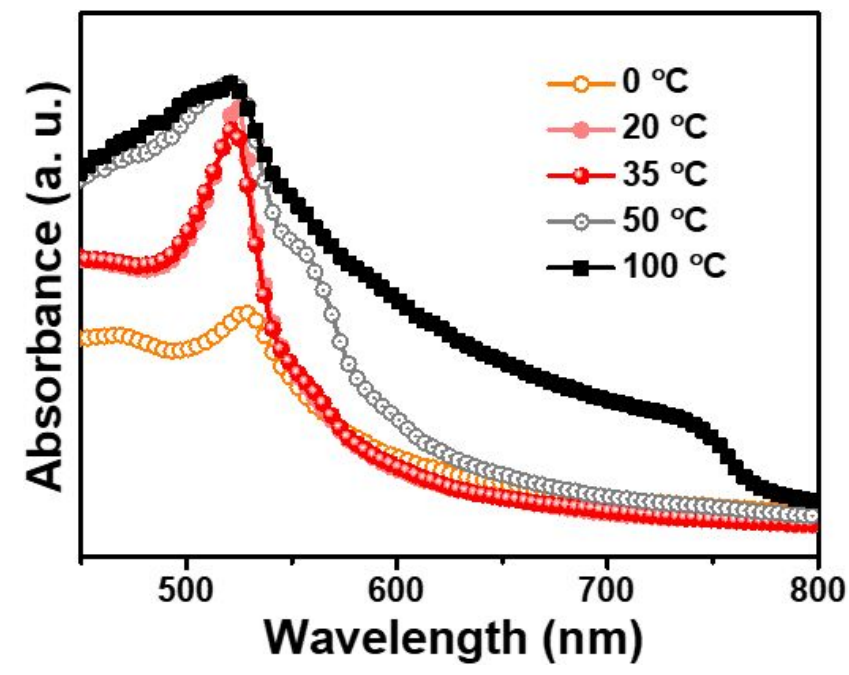

Figure S1. UV-visible absorption spectra of the intermediate films formed at different chlorobenzene temperatures $\left(0,20,35,50\right.$, and $\left.100^{\circ} \mathrm{C}\right)$.

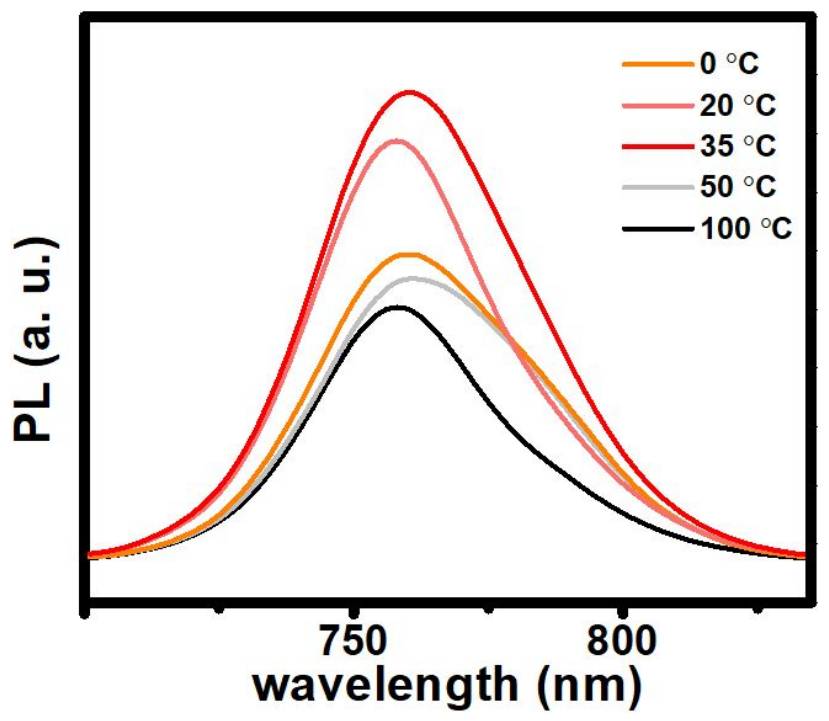

Figure S2. Steady-state photoluminescence of perovskites on tin oxide layer. 


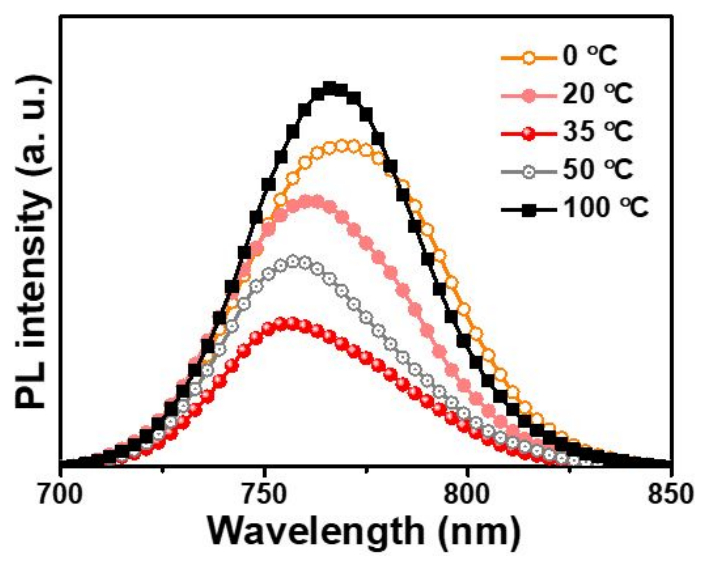

Figure S3. Photoluminescence quenching of perovskites on tin oxide layer.

(a)
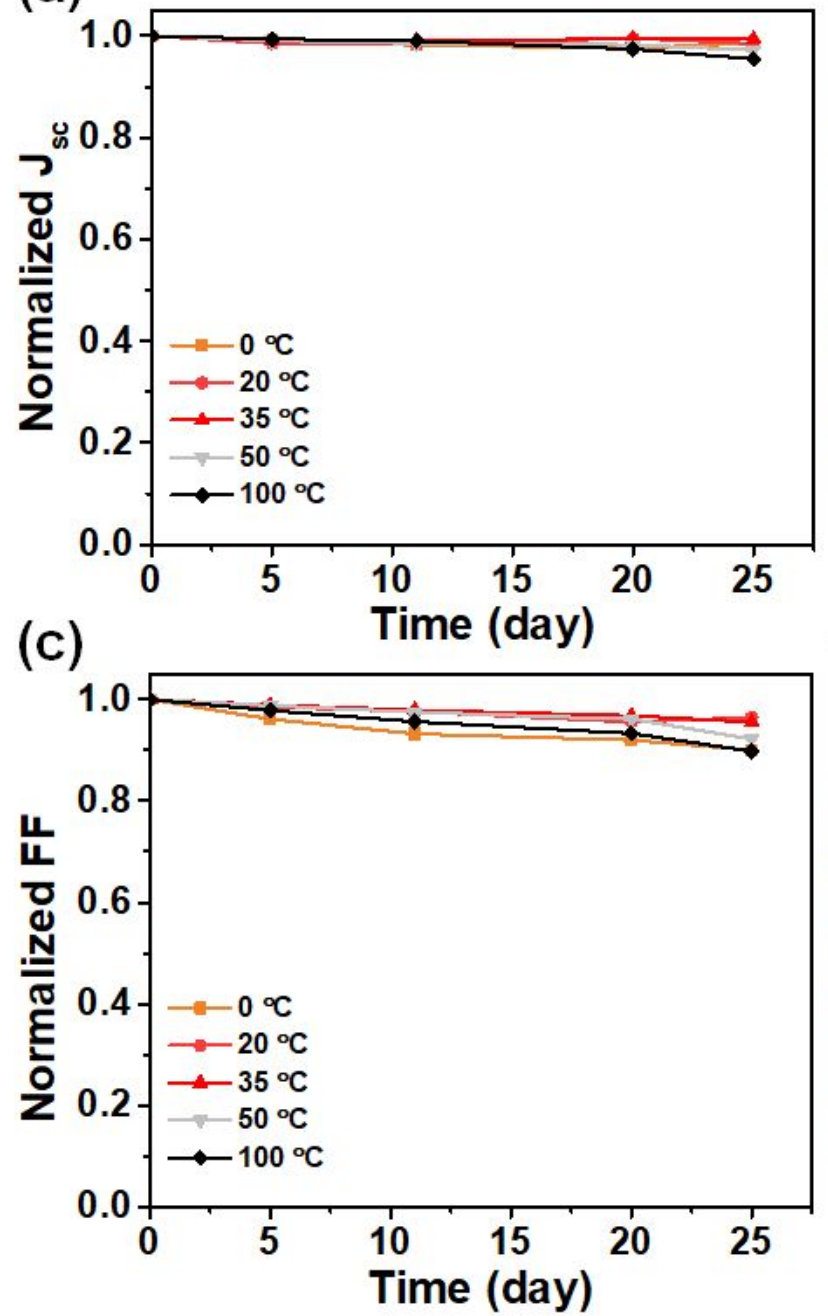

(b)

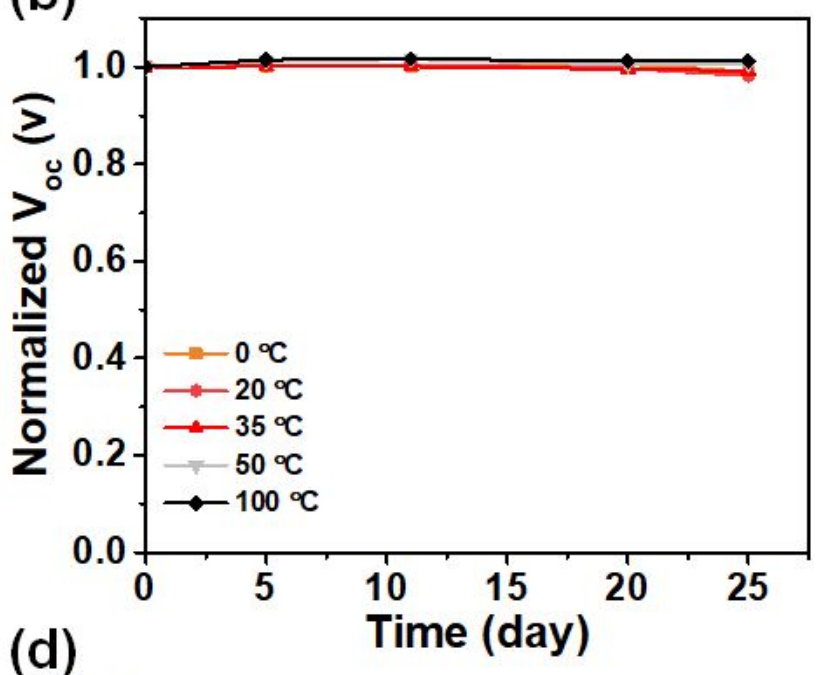


Figure S4. Stability tests of each device fabricated at various temperatures: (a) short-circuit current, (b) open-circuit voltage, (c) fill factor, (d) power conversion efficiency. 
Table S1 The thickness of perovskite films formed at different chlorobenzene temperatures (0, 20, 35,50 , and $100{ }^{\circ} \mathrm{C}$ ).

\begin{tabular}{cc}
\hline Temperature $\left({ }^{\circ} \mathrm{C}\right)$ & Thickness $(\mathrm{nm})$ \\
\hline 0 & 300 \\
20 & 390 \\
35 & 433 \\
50 & 470 \\
100 & 580 \\
\hline
\end{tabular}

Table S2 The average photovoltaic parameters of full devices with different chlorobenzene temperatures

\begin{tabular}{ccccc}
\hline Temperature $\left({ }^{\circ} \mathrm{C}\right)$ & $\begin{array}{c}J_{\mathrm{SC}} \\
\left(\mathrm{mA} / \mathrm{cm}^{2}\right)\end{array}$ & $\begin{array}{c}V_{\mathrm{OC}} \\
(\mathrm{V})\end{array}$ & $\begin{array}{c}\mathrm{FF} \\
(\%)\end{array}$ & $\begin{array}{c}\text { PCE } \\
(\%)\end{array}$ \\
\hline \multirow{2}{*}{0} & 22.10 & 1.11 & 70.64 & 17.33 \\
& $( \pm 0.75)$ & $( \pm 0.02)$ & $( \pm 1.89)$ & $( \pm 0.97)$ \\
20 & 23.14 & 1.13 & 73.04 & 19.23 \\
& $( \pm 0.55)$ & $( \pm 0.02)$ & $( \pm 2.23)$ & $( \pm 0.66)$ \\
35 & 23.73 & 1.14 & 74.83 & 20.25 \\
& $( \pm 0.25)$ & $( \pm 0.01)$ & $( \pm 1.63)$ & $( \pm 0.36)$ \\
50 & 22.55 & 1.13 & 71.03 & 18.34 \\
& $( \pm 0.41)$ & $( \pm 0.01)$ & $( \pm 2.58)$ & $( \pm 0.57)$ \\
& 21.58 & 1.10 & 67.77 & 16.49 \\
& $( \pm 0.89)$ & $( \pm 0.01)$ & $( \pm 3.98)$ & $( \pm 1.18)$ \\
\hline
\end{tabular}

